We sought to determine the cost-effectiveness of each UK guideline from an NHS perspective, plus alternatives, using prospective data.

All patients with a new HIV diagnosis attending an ambulatory HIV clinic, plus a sample of those with known HIV were approached; and offered a symptom questionnaire, chest radiograph (CXR), tuberculin skin test (TST), blood interferon gamma release assay (IGRA) and induced sputum for mycobacterial culture (IS). The uptake and results were used to calculate the costeffectiveness of thirty different testing strategies using univariate, multivariate and probabilistic sensitivity analyses (PSA).

219 subjects, representative of the total clinic population, took part. $73 \%$ were male, $28 \%$ black African and $95 \%$ on antiretroviral therapy (ART). During testing, 2 cases $(0.9 \%)$ of subclinical TB and $14(6 \%)$ of LTBI were detected. Half the patients with LTBI completed preventive treatment. Over a median of 28 months follow up, no new cases of active TB were identified.

When compared to no testing, only three of the thirty strategies were below the maximum NICE threshold for cost-effectiveness $<£ 30,000 / \mathrm{QALY}$ gained. Testing black Africans with just TST or IGRA cost $£ 23,429 / \mathrm{QALY}$ and $£ 28,971$ /QALY respectively, whilst testing black Africans plus those from countries with a TB incidence of $>20 / 100,000$ ('middle incidence', MI) cost $£ 25,218$ /QALY and $£ 32,410$ /QALY using TST alone or IGRA alone respectively. NICE, BHIVA, or more extensive strategies, were not cost-effective. (Table)

Using PSA, no testing was most likely cost-effective up to $£ 30,000 /$ QALY.

In a contemporary HIV population with very high uptake of ART, neither current UK guideline is cost-effective. Testing black Africans, or black Africans and people from middle TB incidence countries appear at best marginally cost-effective. Future UK guidance needs to reflect changing health demographics, improved outcomes for people in HIV care, and clinical pragmatism.

\section{S89 THE USE OF TUBERCULOSIS CHEMOPROPHYLAXIS IN PATIENTS OF RENAL REPLACEMENT THERAPY}

${ }^{1} \mathrm{~N}$ Ahern, ${ }^{2} \mathrm{H}$ Jarvis, ${ }^{2} \mathrm{R}$ Charif, ${ }^{2} \mathrm{OM}$ Kon. ${ }^{1}$ Imperial College London, London, UK; ${ }^{2}$ Imperial College NHS Healthcare Trust, London, UK

\subsection{6/thoraxjnl-2016-209333.95}

Introduction Individuals with end stage renal disease (ESRD) undergoing renal replacement therapy (RRT) are at increased risk of tuberculosis (TB). Timely identification and treatment of latent TB infection (LTBI) reduces the risk of progression to active disease. Diagnosing LTBI is challenging in ESRD as standard tests, such as the tuberculin skin test (TST) and Interferon Gamma Release Assay (IGRA) are less reliable. Although TB guidelines exist for ESRD they acknowledge a lack of evidence base and are limited in their scope.

This study aimed to establish the current LTBI screening and treatment practice in patients of RRT in a central London teaching hospital with the hypothesis that there would be a varied approach with overall low levels of screening.

Methods New starters on haemodialysis (HD) in the year 2010 were identified from computerised renal databases and information collected on; demographics, renal diagnosis, co-morbidities, dialysis attributes, TB risk factors, screening methods and LTBI treatment. All patients were followed for a period of 5 years to establish the rate of active TB after commencing RRT. Screening was considered to have taken place if any of the following were performed irrespective of symptoms of active disease; TST, IGRA, radiography specifically to investigate for TB or documented risk stratification.

Results Of the 331 eligible patients only 77 (23.2\%) received screening. In those who were screened, 13 (16.9\%) were diagnosed with active TB equating to an incidence of 3927/100,000, 37 (48.1\%) with latent TB and 27 (35.1\%) with neither. Risk factor stratification was the commonest modality of LTBI identification although there were a wide variety of approaches. Chemoprophylactic treatment regimes were non-standardised and often based on clinical experience rather than guidelines. Of those with active infection, disease developed most commonly within the first year of starting HD.

Conclusion High rates of active TB occur mainly within the first year of RRT. There is a lack of a uniform approach to detecting and treating LTBI in this population currently. Risk stratification and the use of immunological tests may offer the most sensitive approach to screening ESRD and within the first year of RRT.

\section{S90 THE NATURE AND DURATION OF SYMPTOMS AND TIME TO STARTING TREATMENT COMPARING OLDER WITH YOUNGER PULMONARY TUBERCULOSIS PATIENTS}

${ }^{1} \mathrm{~A}$ Abbara, ${ }^{2} \mathrm{E}$ Hardman, ${ }^{3} \mathrm{SM}$ Collin, ${ }^{4} \mathrm{OM}$ Kon, ${ }^{1} \mathrm{Z}$ Mahomed, ${ }^{5} \mathrm{JAL}$ Sullivan, ${ }^{5} \mathrm{~K}$ Buell, ${ }^{6} \mathrm{~T}$ Hansel, ${ }^{1} \mathrm{~T}$ Corrah, ${ }^{1} \mathrm{RN}$ Davidson. ' London North West Healthcare NHS Trust, London, UK; ${ }^{2}$ University College Hospital, London, UK; ${ }^{3}$ University of Bristol, Bristol, UK; ${ }^{4}$ Imperial Healthcare NHS Trust, London, UK; ${ }^{5}$ Imperial College, London, UK; ${ }^{6} \mathrm{NHLI}$, Imperial College, London, UK

\subsection{6/thoraxjnl-2016-209333.96}

Introduction and objectives In 2013, Public Health England (PHE) reported that 39\% of pulmonary tuberculosis (TB) patients $>65$ years old started treatment $>4$ months after symptom onset compared to $25 \%$ of patients aged $15-44$ years. A longer symptomatic period, particular if smear positive, may result in increased transmission and poorer treatment outcomes. We investigated age-related differences in symptom duration and time to starting $\mathrm{TB}$ treatment in a large UK cohort of $\mathrm{TB}$ patients.

Methods The cohort comprised patients on the London TB register (LTBR) who were treated at Northwick Park Hospital between 2002-2015. Patients aged >65 years were compared with a random sample of patients aged 18-40 years, with respect to symptoms, symptom duration at presenting to secondary care and time to starting treatment after presenting to secondary care. Results 357 patients over 65 and 517 younger patients were identified. Demographics for the total number of patients are included in Table 1. Data were available for 489 of the younger group and 73 of the older group. 26 of the $>65$ s (35.6\%) and 9 $(1.8 \%)$ of the 65 s were diabetic.

Conclusions This study has identified that a potential cause for a delay in diagnosis in the elderly may be related to the decreased frequency of 'classical' TB symptoms in those $>65$ years of age. Clinicians need to be vigilant despite the lack of these symptoms.

Median duration of symptoms was twice that in older patients versus younger patients (90 days versus 45 ) with a longer time to starting treatment ( 7 days versus 2 ). The total duration (in months) from symptom onset was also higher at 3 months versus 2 months. A higher proportion of older patients (32\%) started treatment greater than 4 months from symptom onset compared to $13.1 \%$ in younger patients. 